\title{
Bit Commitment Using Pseudo-Randomness *
}

\author{
(Extended Abstract)
}

\author{
Moni Naor \\ IBM Almaden Research Center \\ 650 Harry Road \\ San-Jose CA 95120
}

\begin{abstract}
We show how a pseudo-random generator can provide a bit commitment protocol. We also analyze the number of bits communicated when parties commit to many bits simultaneously, and show that the assumption of the existence of pseudo-random generators suffices to assure amortized $O(1)$ bits of communication per bit commitment.
\end{abstract}

\section{Introduction}

A bit commitment protocol is a basic component of many cryptographic protocols. One party, Alice, commits to the other party, Bob, to a bit $b$, in such a way that Bob has no idea what $b$ is. At a later stage Alice can reveal the bit $b$ and Bob can verify that this is indeed the value to which Alice committed. A good way to think about it is as if Alice writes the bit and puts it in a locked box to which only she has the key. She gives the box to Bob (the commit stage) and when the time is ripe, she opens it and Bob knows that the contents were not tampered since the box was at his possession.

Bit commitment has been used for zero knowledge protocols [GMW1], [BCC], identification schemes [FS], Multi party protocols [GMW2], [CDG], and can implement Blum's coin flipping over the phone [B].

A current research program in cryptography is to base the security on as general assumptions as possible. Past successes of the program had been in establishing various

"Part of this work done while author was at UC Berkeley. Research supported by NSF grant CCR $88-13632$ 
primitives on the existence of one-way functions or permutations or on the existence of trapdoor functions. The most general (computational complexity) assumption under which bit commitment was known to be possible is that one way permutations exist [GMW1]. In this paper we show that given any pseudo-random generator, a bit commitment protocol can be constructed. This is a weaker condition, since Yao [Yao] has shown that pseudo-random generators can be based on one-way permutations. A pseudo-random generator is a function that maps a string (the seed) to a longer one, such that if the seed is chosen at random, then the output is indistinguishable from a truly random distribution for all polynomial time machines. Very recently Impagliazzo, Levin and Luby [ILL] have shown that given any one way function (not necessary a permutation), a pseudo-random generator can be constructed. On the other hand, Impagliazzo and Luby [IL] have argued that the existence of one-way functions is a prerequisite for any protocol that must rely on computational complexity. Thus we can conclude that if any computational complexity based cryptography is possible, then bit commitment protocols exist, and so do the protocols that rely on bit commitment, such as zero-knowledge proofs and identification schemes.

What is the communication complexity of a bit commitment protocol (i.e. how many bits must be transferred during the execution of the protocol)? It cannot be the case that only a fixed number of bits will be exchanged during the execution of the protocol, otherwise after the commit stage Bob can guess with non negligible probability what Alice would send in the revealing stage, and can verify that the guess is consistent with what she sent so far and deduce the value of the bit. However, in many applications Alice wants to commit to a collection of bits $b_{1}, b_{2}, \ldots b_{m}$ and they are to be revealed at the same time. These applications include coin flipping over the phone and zero-knowledge protocols such as Impagliazzo and Yung [IY]. Furthermore, Kilian, Micali and Ostrovsky [KMO] have shown that many of the known protocols for zero knowledge can be converted to ones that have this property. Therefore it is desirable to amortize the communication complexity of bit commitment. We show that if $m$ is large enough, at least linear in the security parameter $n$, then Alice can commit to $b_{1}, b_{2}, \ldots b_{m}$ while exchanging only $O(1)$ bits per bit commitment. The total computational complexity of the protocol is the same as the complexity of the protocol for committing to one bit.

In the next section we give formal definitions of the problem and the assumptions. In Section 3 we show how the commit can be implemented using a pseudo-random generator. Section 4 shows how to get the amortized communication complexity down to $O(1)$ per bit. 


\section{Definitions}

A bit commitment protocol consists of two stages:

- The commit stage: Alice has a bit $b$ to which she wishes to commit to Bob. She and Bob exchange messages. At the end of the stage Bob has some information that represents $b$.

- The revealing stage: at the end of which Bob knows $b$.

The protocol must obey the following: For all polynomials $p$ and for large enough security parameter $n$

1. After the commit stage Bob cannot guess $b$ with probability greater than $\frac{1}{2}+\frac{1}{p(n)}$.

2. Alice can reveal only one possible value. If she tries to reveal a different value she is caught with probability at least $1-\frac{1}{p(n)}$.

In defining the properties that a bit commitment protocol must obey we have assumed a scenario where Bob cannot guess $b$ with probability greater than $\frac{1}{2}$ prior to the execution of the commit protocol. In the more general case, Bob has some auxiliary input that might allow him to guess $b$ with probability $q>\frac{1}{2}$. The definition for this case is that as a result the commit stage the advantage that Bob gains in guessing $b$ is less than $\frac{1}{p(n)}$. All the results of this paper hold for the general case.

\section{Pseudo-Random Generators}

Let $m(n)$ be some function such that $m(n)>n$. $G:\{0,1\}^{n} \mapsto\{0,1\}^{m(n)}$ is a a pseudo-random generator if for all polynomials $p$ and all polynomial time algorithms $A$ that attempt to distinguish between outputs of the generator and truly random sequences, except for finitely many $n$ 's:

$$
|\operatorname{Pr}[A(y)=1]-\operatorname{Pr}[A(G(s))=1]|<\frac{1}{p(n)}
$$

where the probabilities are taken over $y \in\{0,1\}^{m(n)}$ and $s \in\{0,1\}^{n}$ chosen uniformly at random.

Remark: We could have defined pseudo-random generators relative to polynomial sized circuits. The results in this paper would be the same in this case.

It is known that if pseudo-random generators exist for any $m(n)>n$, then they exists for all $m$ polynomial in $n$ [GGM]. We can treat the pseudo-random generator as outputting a sequence of unspecified length, of which we can examine only a fixed prefix (whose length is polynomial in $n$, the seed length). 
In the rest of the paper we will assume some pseudo-random generator $G$. Let $n$ be a security parameter which is assumed to have been chosen so that no feasible machine can break the pseudo-random generator for seeds of length $n$. We will use $G_{l}(s)$ to denote the first $l$ bits of the pseudo-random sequence on seed $s \in\{0,1\}^{n}$. $B_{i}(s)$ will be used to denote the $i$ th bit of the pseudo-random sequence on seed $s$.

\section{The Bit Commitment}

A property of pseudo-random sequences that is natural to apply in order to achieve bit commitment is the unpredictability of the next bit: it is known that given the first $m$ bits of a pseudo-random sequence, any polynomial time algorithm that tries to predict the next bit in the sequence has probability smaller than $\frac{1}{2}+\frac{1}{p(n)}$ to succeed for any polynomial $p(n)$. (In fact, Blum and Micali [BM] used this property to define pseudo-randomness and Yao [Yao] gas shown that the two definitions are equivalent.)

As a first attempt, consider the following protocol:

- Commit stage - Alice selects seed $s \in\{0,1\}^{n}$ and sends $G_{m}(s)$ and $B_{m+1}(s) \oplus b$. ( $b$ is the bit Alice is committed to.)

- Reveal stage - Alice sends $s$, Bob verifies that $G_{m}(s)$ is what Alice sent him before and computes $b=B_{m+1}(s) \oplus\left(B_{m+1}(s) \oplus b\right)$

This protocol has the property that Bob cannot guess the bit that Alice commits to before the revealing stage, except with probability smaller than $\frac{1}{2}+\frac{1}{1 \text { poly }}$, because he does not have the power to predict the pseudo-random sequence. On the other hand, Alice might be able to cheat: if she finds two seeds $s_{1}$ and $s_{2}$ such that $G_{m}\left(s_{1}\right)=$ $G_{m}\left(s_{2}\right)$, but $B_{m+1}\left(s_{1}\right) \neq B_{m+1}\left(s_{2}\right)$, then she can reveal any bit she wishes (by sending $s_{1}$ or $\left.s_{2}\right)$. There is nothing in the definition of pseudo-random generators that forbids the existence of such pairs. Furthermore, given any pseudo-random generator $G$, one can construct another pseudo-random generator $G^{\prime}$ that has such pairs.

There is no way to force Alice to stick to one seed, since there may be two seeds that yield the same sequence. However, what the following protocol does is to force Alice to stick to the same sequence, or she will be caught with high probability.

\section{Bit Commitment Protocol}

- Commit stage -

1. Bob selects a random vector $\vec{R}=\left(r_{1}, r_{2}, \ldots r_{3 n}\right)$ where $r_{i} \in\{0,1\}$ for $1 \leq i \leq 3 n$ and sends it to Alice. 
2. Alice selects a seed $s \in\{0,1\}^{n}$ and sends to Bob the vector $\vec{D}=$ $\left(d_{1}, d_{2}, \ldots d_{3 n}\right)$ where

$$
d_{i}= \begin{cases}B_{i}(s) & \text { if } r_{i}=0 \\ B_{i}(s) \oplus b & \text { if } r_{i}=1\end{cases}
$$

- Reveal stage - Alice sends $s$ and Bob verifies that for all $1 \leq i \leq 3 n$, if $r_{i}=0$ then $d_{i}=B_{i}(s)$, and if $r_{i}=1$ then $c_{i}=B_{i}(s) \oplus b$.

This protocol maintains the property that Bob learns nothing about the bit $b$, otherwise we claim that Bob has the power to distinguish between outputs of the pseudo-random generator and truly random strings: if Alice had chosen a truly random sequence instead of a pseudo-random sequence, then Bob would not have learned anything about $b$, since all vectors $\vec{D}$ are equally likely, no matter what $b$ is. If there exists a polynomial time Bob (call him Bob') that can learn something about $b$ when Alice uses a pseudo-random sequence, then $\mathrm{Bob}^{\prime}$ can be used to construct a distinguisher between outputs of $G$ and truly random sequences. Given a sequence $x$, run the commit stage of the protocol with Alice and $\mathrm{Bob}^{\prime}$, where Alice commits to a random $b$ and instead of a creating a pseudo-random sequence uses $x$. Let Bob' guess $b$. If he guesses correctly decide that $x$ is pseudo-random, otherwise decide that $x$ is truly random. The difference in the probability of deciding that the sequence is pseudo-random between a random sequence and a pseudo-random sequence is equal to the advantage Bob' has of guessing $b$ in case $x$ is a pseudo-random sequence.

How can Alice cheat? Her only chance to cheat is if there exist two seeds $s_{1}$ and $s_{2}$ such that $G_{3 n}\left(s_{1}\right)$ and $G_{3 n}\left(s_{2}\right)$ agree in all positions $i$ where $r_{i}=0$, and totally disagree in all positions $i$ where $r_{i}=1$. We say that such a pair fools $\vec{R}$.

Claim 3.1 The Probability that there exists a pair of seeds $s_{1}$ and $s_{2}$ that fools $\vec{R}$ is at most $2^{-n}$, where the probability is taken over the choices of $\vec{R}$.

Proof: If a pair $s_{1}, s_{2}$ fools $\vec{R}$, then we know that $r_{i}=B_{i}\left(s_{1}\right) \oplus B_{i}\left(s_{2}\right)$. Therefore, a pair $s_{1}$ and $s_{2}$ fools exactly one $\vec{R}$. There are $2^{2 n}$ pairs of seeds and $2^{3 n}$ vectors $\vec{R}$. Hence the probability that there exists a pair that can fool the $\vec{R}$ that Bob chose is at most $\frac{2^{2 n}}{2^{3 n}}=2^{-n}$.

We can summarize by

Theorem 3.1 If $G$ is a pseudo-random generator, then the bit commitment protocol presented obeys the following: For all polynomials $p$ and for large enough security parameter $n$

1. After the commit stage Bob cannot guess $b$ with probability greater than $\frac{1}{2}+\frac{1}{p(n)}$

2. Alice can reveal only one possible bit, except with probability less than $\frac{1}{p(n)}$ 


\section{Efficient Commit to Many Bits}

The protocol given in the previous section has communication cost of $O(n)$ bits. If Alice wants to commit to many bits $b_{1}, b_{2}, \ldots b_{m}$ which she will reveal simultaneously, then she can do better.

The idea is to use many bits to force Alice to stick to one sequence and use that sequence to commit to many bits.

Suppose we implement a protocol similar to the one in the previous section, but for the part of the pseudo-random sequence that Bob request to see its Xor with $b$ we give its bit-wise Xor with $b_{1}, b_{2}, \ldots b_{m}$. Alice might be able to alter one of the $b_{i}$ 's, since it is enough that there exists a pair of seeds that agree on all the bits but one.

We will prevent this from happening by using error correcting codes with large distance between code words. Let $C \subset\{0,1\}^{q}$ be a code of $2^{m}$ words such that the hamming distance between any $c_{1}, c_{2} \in C$ is at least $\epsilon \cdot q$. We will also require that there will be an efficiently computable function $E:\{0,1\}^{m} \mapsto\{0,1\}^{a}$ for mapping words in $\{0,1\}^{m}$ to $C$.

What are the requirement from the code? As we shall see, $\log \frac{1}{1-\epsilon} \cdot q$ must be at least $3 n$, and we want $q / m$ to be a fixed constant. Such codes exist, and specifically the Justesen code is a constructive example [Ju]. For the amortization to work it sufficient that $m$ be linear in $n$.

For a vector $\vec{R}=\left(r_{1}, r_{2}, \ldots r_{k}\right)$ with $r_{i} \in\{0,1\}$ and with exactly $q$ indices $i$ such that $r_{i}=1$ let $G_{\vec{R}}(s)$ denote the vector $\vec{A}=\left(a_{1}, a_{2}, \ldots a_{q}\right)$ where $a_{i}=B_{j(i)}(s)$ and $j(i)$ is the index of the $i$ th 1 in $\vec{R}$. If $e_{1}, e_{2} \in\{0,1\}^{q}$, then $e_{1} \oplus e_{2}$ denotes the bitwise Xor of $e_{1}$ and $e_{2}$.

\section{Commit to Many Bits Protocol}

Alice commits to $b_{1}, b_{2}, \ldots b_{m}$.

- Commit stage -

1. Bob selects a random vector $\vec{R}=\left(r_{1}, r_{2}, \ldots r_{2 q}\right)$ where $r_{i} \in\{0,1\}$ for $1 \leq i \leq 2 q$ and exactly $q$ of the $r_{i}$ 's are 1 and sends it to Alice

2. Alice computes $c=E\left(b_{1}, b_{2}, \ldots b_{m}\right)$. Alice select a seed $s \in\{0,1\}^{n}$ and sends to Bob e $=c \oplus G_{\bar{R}}(s)$ (the bitwise Xor of $G_{\vec{R}}(s)$ and $c$ ), and for each $1 \leq i \leq 2 q$ such that $r_{i}=0$ she sends $B_{i}(s)$.

- Reveal stage - Alice sends $s$ and $b_{1}, b_{2}, \ldots b_{m}$. Bob verifies that for all $1 \leq$ $i \leq 2 q$ such that $r_{i}=0$ Alice had sent the correct $B_{i}(s)$ and computes $c=$ $E\left(b_{1}, b_{2}, \ldots b_{m}\right)$ by computing $G_{\vec{R}}(s)$ and verifies that $e=c \oplus G_{\mathbb{R}}(s)$ 
As in the previous section, Bob can learn nothing about any of the $b_{i}$ 's. When can Alice cheat? She can cheat if there exists a pair of seeds $s_{1}$ and $s_{2}$ that agree on all the indices that $\vec{R}$ has a 0 , and there exist two different sequences $b_{1}, b_{2}, \ldots b_{m}$ and $b_{1}^{\prime}, b_{2}^{\prime}, \ldots b_{m}^{\prime}$ such that $G_{R}\left(s_{1}\right) \oplus E\left(b_{1}, b_{2}, \ldots b_{m}\right)=G_{R}\left(s_{2}\right) \oplus E\left(b_{1}^{\prime}, b_{2}^{\prime}, \ldots b_{m}^{\prime}\right)$. We will say that $s_{1}$ and $s_{2}$ fool $\vec{R}$ in this case.

Claim 4.1 For any pair of seeds $s_{1}$ and $s_{2}$, the Probability that it fools $\vec{R}$ is at most $\left(1-\frac{\varepsilon}{2}\right)^{q}$, where the probability is taken over the choices of $\vec{R}$.

Proof: If $s_{1}$ and $s_{2}$ can fool any $\vec{R}$, then the hamming distance between $G_{2 q}\left(s_{1}\right)$ and $G_{2 q}\left(s_{2}\right)$ must be at least $\epsilon q$, since $G_{\vec{R}}\left(s_{1}\right) \oplus e=c_{1}$ and $G_{\vec{R}}\left(s_{2}\right) \oplus e=c_{2}$ for two different code words $c_{1}$ and $c_{2}$ whose distance is at least $\epsilon q$. Therefore, the probability that the indices $i$ for which $r_{i}=0$ will hit only the indices where $G_{2 q}\left(s_{1}\right)$ and $G_{2 q}\left(s_{2}\right)$ agree is at most $\left(\frac{2 q-\epsilon q}{2 q}\right)^{q}=\left(1-\frac{\epsilon}{2}\right)^{q}$.

If $\log \frac{1}{1-\epsilon} \cdot q>3 n$, then for at most $2^{-n}$ of the vectors $\vec{R} \in\{0,1\}^{2 q}$ there is a pair of seeds $s_{1}$ and $s_{2}$ that fool $\vec{R}$. Therefore, Alice's chances of being able to alter any bit are at most $2^{-n}$.

The number of bits exchanged in the protocol is $O(q)$, and when amortized over $m$ bits it is $O(q / m)$ which is $O(1)$, since $C$ is a good code. The dominant factor in the computational complexity of the protocol is that of $G$. Alice has to produce a pseudorandom sequence of length $2 q$ which is $O(n)$. This is similar to the requirement in the one bit commitment.

We can summarize by

Theorem 4.1 If $G$ is a pseudo-random generator, then the many bit commitment protocol presented obeys the following: For all polynomials $p$ and for large enough security parameter $n$

1. After the commit stage Bob cannot guess any $b_{i}$ with probability greater than $\frac{1}{2}+\frac{1}{p(n)}$, even when told $b_{1}, b_{2}, \ldots, b_{i-1}, b_{i+1}, \ldots b_{n}$

2. For all $1 \leq i \leq m$, Alice can reveal only one possible value for $b_{i}$, except with probability less than $\frac{1}{p(n)}$

Kilian has suggested a different method for amortizing the communication complexity: commit to a seed $s$ by committing to each of its bits separately and then commit to $b_{1}, b_{2} \ldots b_{m}$ by providing its Xor with the pseudo-random sequence generated by $s$. However, in this method the amortization starts only when $m$ is at least $n^{2}$. 


\section{Conclusions}

We have shown how to construct bit commitment protocols from pseudo-random generators and have shown how bit commitment to many bits can be implemented very efficiently. Thus, various Zero-Knowledge protocols can be implemented with low complexity.

In both protocols we have presented, Bob selects a random $\vec{R}$, and we have argued that almost all the $\vec{R}$ 's are good. Therefore if there is a trusted party at some point in time (say the protocol designer), it can choose $\vec{R}$ and the same $\vec{R}$ will be used in all executions of the protocol.

\section{References}

[B] M. Blum, Coin Flipping by Telephone, Proc. 24th IEEE Compcon, 1982, pp. 133-137.

[BM] M. Blum, S. Micali How to Generate Cryptographically Strong Sequences of Pseudo-Random Bits, Siam J. on Computing, vol 13, 1984, pp 850-864.

[BCC] G. Brassard, D. Chaum, C. Crépeau, Minimum Disclosure Proofs of Knowledge, Journal of Computer and System Sciences 37 (1988), pp. 156-189.

[CDG] D. Chaum, I. Damgård and J. van de Graaf, Multiparty Computations Ensuring Secrecy of each Party's Input and Correctness of the Output, Proc. of Crypto 87.

[FS] A. Fiat and A. Shamir, How to prove yourself, Proc. of Crypto 86, pp. 641-654.

[GGM] O. Goldreich, S. Goldwasser and M. Micali, How to construct random functions, Journal of the ACM, vol 33, 1986, pp. 792-807.

[GMW1] O. Goldreich, M. Micali, A. Wigderson, Proofs that yield nothing but their validity and a methodology of cryptographic protocol design, Proc. 27th Symposium on Foundations of Computer Science, 1986, pp 174-187.

[GMW2] O. Goldreich, M. Micali, A. Wigderson, How to play any mental game, Proc. 19th Symposium on Theory of Computing, 1987, pp. 218-229.

[IL] I. Impagliazzo and M. Luby, One-way functions are essential to computational based cryptography, Proc. 21st Symposium on Theory of Computing, 1989. 
[ILL] I. Impagliazzo, L. Levin and M. Luby, Pseudo-random generation from oneway functions, Proc. 21st Symposium on Theory of Computing, 1989.

[IY] R. Impagliazzo and M. Yung, Direct Zero-Knowledge Protocols, Crypto 87.

[Ju] J. Justesen, A class of constructive asymptotically good algebraic codes, IEEE trans. on Information theory 18 (1972) 652-656.

[KMO] J. Kilian, S. Micali and R. Ostrovsky, Simple non-interactive zero-knowledge proofs, Crypto 89.

[Yao] A. C. Yao, Theory and Applications of Trapdoor Functions, Proc. 23rd Symposium on Foundations of Computer Science, 1982, pp 80-91. 